\title{
Mastopatía diabética. Un diagnóstico poco habitual
}

\author{
Juan Antonio Pérez P, Cristián Carrasco L. \\ Diabetic mastopathy. \\ Report of one case
}

Diabetic mastopathy is a rare condition that occurs in type I diabetic patients and that may mimic a breast cancer due to its intense fibrosis and inflammation. We report a 34 years old female consulting for a firm, painless and mobile retroareolar breast lump measuring $3.5 \times 4 \mathrm{~cm}$, that lead to the suspicion of a breast cancer. Mammography, mammary ultrasound and fine needle cytology were unspecific. A core biopsy confirmed the diagnosis of diabetic mastopathy. The patient continues under periodical assessments (Rev Méd Chile 2003; 131: 1305-08 ).

(Key Words: Breast diseases; Diabetes mellitus, juvenile-onset; Mammary neoplasms; Mammography)

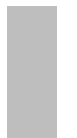

Recibido el 29 de mayo, 2003. Aceptado el 1 de septiembre, 2003.

Institutos de Cirugía e Histopatología y Patología, Facultad de Medicina, Universidad Austral de Chile.

Servicios de Cirugía y Anatomía Patológica, Hospital Clínico Regional de Valdivia.

L a mastopatía diabética es una lesión fibroinflamatoria de la mama, muy infrecuente, que característicamente se presenta en mujeres premenopáusicas con diabetes mellitus tipo I, de larga evolución y con múltiples complicaciones microvasculares $^{1-4}$. En el hombre es excepcional ${ }^{5}$. La etiopatogenia es desconocida y no predispone al desarrollo de cáncer mamario o linfoma. Clínicamente se presenta como masa mamaria única o múltiple, en una o en ambas mamas, de rápido crecimiento, habitualmente en localización subareolar, de consistencia firme o dura, indolora, móvil y sin compromiso de la piel que la recubre. Estas características sugieren la sospecha de malignidad por lo cual es fundamental excluir esta posibilidad. La mamografía, ecotomografía mama-

Correspondencia a: Dr. Juan Antonio Pérez P. Arturo Günther 1664, Valdivia. Fono: 063214883.

E-mail: jperez@uach.cl ria $\mathrm{y}$ resonancia nuclear magnética ${ }^{6-8}$ pueden contribuir al diagnóstico, sin embargo no muestran imágenes específicas por lo cual para establecer con certeza la naturaleza biológica de la lesión es necesario llegar al estudio histopatológico.

El conocimiento de las características de esta entidad clínica puede ahorrar múltiples e injustificados procedimientos quirúrgicos en los enfermos que padecen esta condición, los que a menudo presentan un estado de deterioro significativo. Comunicamos un caso clínico de mastopatía diabética y discutimos algunos aspectos de esta interesante enfermedad.

\section{Caso Cúnico}

Mujer de 34 años, portadora de diabetes mellitus tipo I desde los 15 años de edad, que evolucionó 
con nefropatía diabética y luego insuficiencia renal diagnosticada a los 28 años, llegando a hemodiálisis trisemanal a partir de los 32 años. Además desarrolló otras complicaciones, destacando retinopatía, neuropatía y gastropatía diabética, hipertensión arterial y anemia.

El 25 de noviembre de 2002 descubrió en forma incidental una dureza en la mama derecha, motivo por el cual acudió a la Unidad de Patología Mamaria. Evaluada el 10 de diciembre de 2002, relataba antecedentes de cáncer mamario familiar en tía materna y en una prima, menarquia a los 20 años, nuligesta, no usuaria de anticonceptivos, sin patología tiroidea. En el examen físico destacó mamas de tamaño normal, simétricas, con zona indurada de 3,5 x $4 \mathrm{~cm}$ en la región retroareolar insinuada hacia los cuadrantes externos de la mama derecha, mal delimitada, indolora, móvil. En los territorios nodales axilares y supraclaviculares no se palparon linfoadenopatías sospechosas. Se diagnosticó tumor de mama derecha ¿cáncer mamario?

Se solicitó una mamografía que informó tejido fibroglandular de densidad aumentada, destacando en la región retroareolar derecha una imagen nodular de mayor densidad, de bordes parcialmente definidos, que medía $3,5 \mathrm{~cm}$ en su diámetro mayor. BI-RADS 0 (Figura 1). Era indispensable estudio ecotomográfico. La ecotomografía mamaria mostró en la mama derecha, hacia la unión de los cuadrantes externos, adyacente al pezón, una

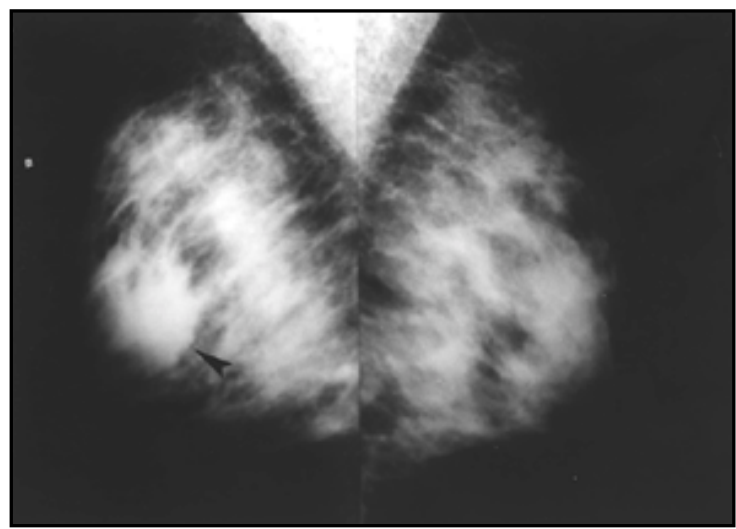

FIgURA 1. Mamografía bilateral centrolateral muestra tejido fibroglandular abundante con aumento localizado de la densidad en región retroareolar derecha (punta de flecha). imagen nodular mal definida, de 4,8 x 2,7 cm, hipoecogénica, que proyectaba una sombra acústica posterior, con características sospechosas de malignidad. Se sugirió estudio histológico (Figura 2). La citología por punción aspirativa identificó escasos grupos de células epiteliales normotípicas. El 16 de diciembre 2002 se realizó biopsia core resultando difícil extraer en forma expedita cilindros de tejido tumoral, debido a que la firmeza del tumor impedía desplazar con facilidad la aguja. El estudio histopatológico reveló tejido mamario con ductos normotípicos, con expansión del colágeno con aspecto de queloide. Entre el colágeno, se identifican miofibroblastos con un foco de fibroblastos reactivos (Figuras 3 y 4). Se concluyó que eran hallazgos histológicos compatibles con mastopatía diabética.

Considerando los hallazgos y antecedentes de la paciente se resolvió realizar nueva evaluación clínica, imagenológica e histológica en un plazo de 4 meses, constatándose luego de ese período que la lesión no modificó sus características cumpliendo así con los criterios para ser catalogada como mastopatía diabética. Con dicho diagnóstico se decidió mantenerla en controles periódicos.

\section{COMENTARIO}

Las complicaciones microvasculares de la diabetes mellitus que incluyen neuropatía, nefropatía y

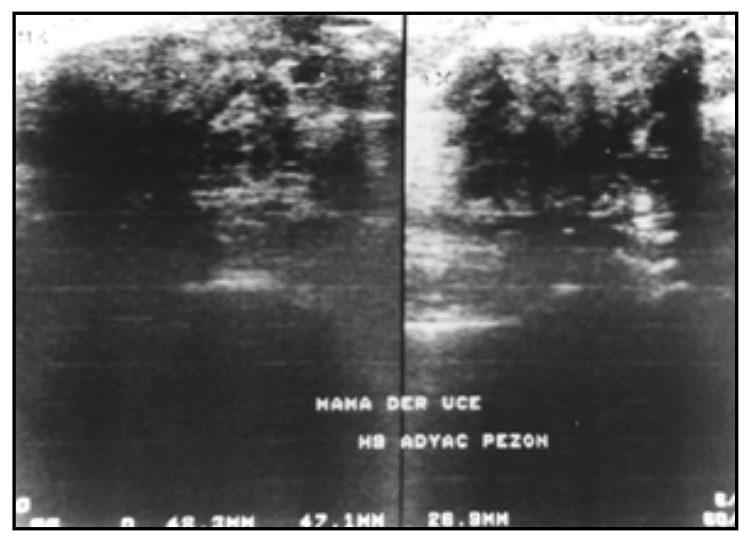

FIgURA 2. En la ecotomografía de la mama derecha se observa una imagen nodular irregular, hipoecogénica, con sombra acústica posterior. 


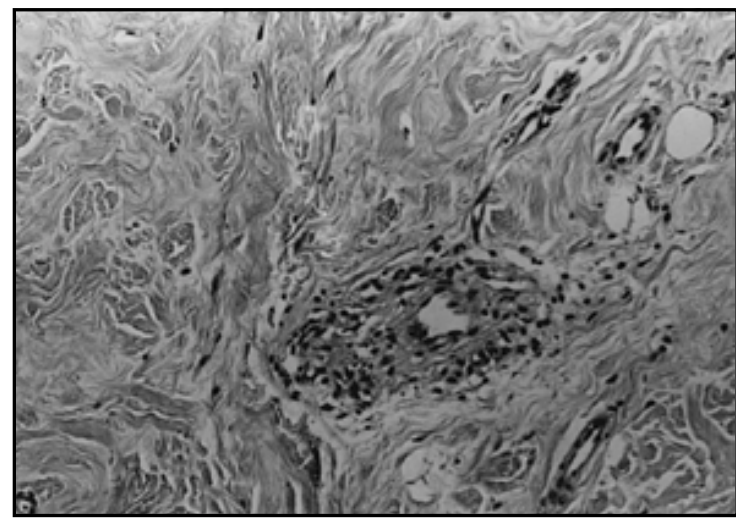

FIgura 3. Se observa tejido fibroso de aspecto queloideo y en la porción central se advierte un vaso sanguíneo con gran cantidad de linfocitos en su periferia. H-E $40 \mathrm{x}$.

retinopatía diabética son bien conocidas y se relacionan con la dilatada evolución de la enfermedad. Una complicación poco corriente es la mastopatía diabética, también denominada enfermedad mamaria diabética fibrosa o lobulitis linfocitaria. Fue descrita por primera vez en el año 1984 por Soler y Khardori ${ }^{10}$ en una serie de 12 pacientes. Su frecuencia es difícil de precisar, aunque se estima que representa menos de $1 \%$ de las lesiones benignas de la mama, y que aparece en alrededor de $13 \%$ de las diabéticas insulinodependientes de larga evolución ${ }^{4}$.

El origen de la mastopatía diabética es desconocido. Se postula que la lesión se originaría por una reacción autoinmune secundaria a una acumulación anormal de matriz extracelular siendo ésta una de las manifestaciones de los efectos de la hiperglicemia en el tejido conectivo. La glicosilación y el aumento de las uniones intermoleculares producirían un colágeno resistente a la degradación ${ }^{11}$.

El diagnóstico puede sospecharse por la historia clínica, en pacientes premenopáusicas con diabetes mellitus tipo I de larga data que desarrollan una masa mamaria indolora, de tamaño variable entre 2 a $10 \mathrm{~cm}$, en una o en ambas mamas. En el examen físico la masa puede simular un cáncer de mama. La mamografía, en general, tiene poco rendimiento para orientar el diagnóstico. Las lesiones son poco visibles, puede observarse un aumento localizado de la densidad,

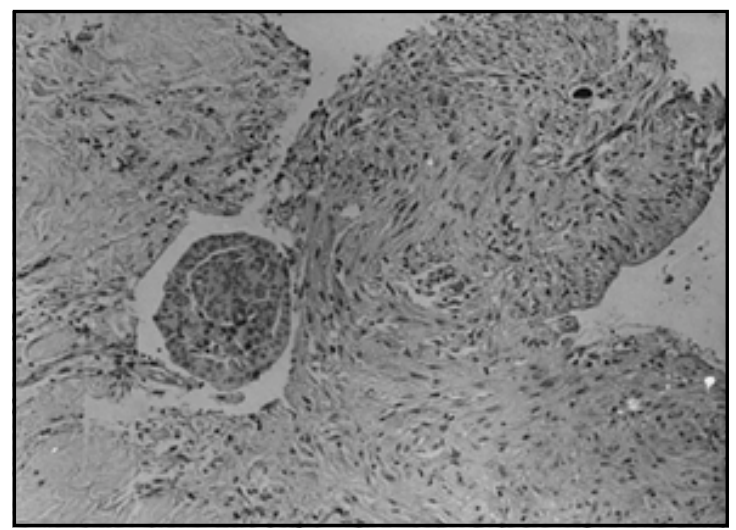

FIgURA 4. Tejido mamario con fibroblastos reactivos y miofibroblastos. Se aprecia conducto mamario retraído, sin atipias. H-E $20 \mathrm{x}$.

sin distinguirse masas, espiculaciones o calcificaciones $^{2}$, no obstante lo más usual es que sólo se aprecie tejido mamario denso y heterogéneo, la mayoría de las veces asimétrico. El ultrasonido es el examen complementario más útil para evaluar una masa mamaria sospechosa de mastopatía diabética $^{12}$ ya sea palpable o subclínica, constituyendo en este último caso una herramienta valiosa para guiar la obtención de la biopsia. Muestra lesiones hipoecogénicas de márgenes irregulares, con marcada sombra acústica posterior, que es más pronunciada que la que se observa en los cánceres mamarios.

El diagnóstico histológico es indispensable cuando existe sospecha clínica y radiológica de mastopatía diabética. La interrogante se plantea sobre la forma de obtención de material para la biopsia. Se ha sugerido que el diagnóstico podría obtenerse mediante citología por punción aspirativa y también utilizar este método para el seguimiento de las pacientes, sin embargo el procedimiento resulta útil en no más de la mitad de los casos, al no lograr obtener suficiente muestra para estudio, por tratarse de masas duras y firmes3. Por tal motivo, para establecer el diagnóstico definitivo se considera más adecuado la realización de biopsias que pueden ser abiertas 0 de preferencia biopsia core $2,7,8$.

La resección amplia de una o varias lesiones puede resultar en una cirugía innecesaria y antiestética. Cuando una masa se reseca quirúrgicamen- 
te se ha reportado que alrededor de $60 \%$ recidivan 0 aparecen en la mama contralateral.

El examen microscópico revela la presencia de estroma colágeno denso de aspecto queloideo con áreas de fibroblastos reactivos, algunos de aspecto epiteloideo y con características de miofibroblastos. Se observa además infiltrado inflamatorio compuesto por linfocitos maduros, predominantemente B, de distribución perivascular, así como alrededor de lóbulos y conductos, raramente se forman centros germinales. La mayor parte de las veces concurren todas estas alteraciones, pero en ocasio-

\section{REFERENCIAS}

1. Kudva YC, Reynolds C, O’Brien T, Powell C, Oberg AL, CRotTy TB. Diabetic mastopathy or sclerosing lymphocytic lobulitis, is strongly associated with type 1 diabetes. Diabetes Care 2002; 25: 121-6.

2. Camuto PM, MD, Zetrenne E, Ponn T. Diabetic mastopathy. A report of 5 cases and a review of the literature. Arch Surg 2000; 135: 1190-3.

3. Zimmerui L, Yurtsever H, Conen D, Truninger K. A diabetic breast lump. Lancet 2001; 357: 1670.

4. Zaragoza C, García Ja, Mauir F, Vázquez JL. Diabetic mastopathy; source of confusion with breast cancer. Med Clin (Barc) 2001; 117: 718-9.

5. SuÁrez S, SÁnchez N. Diabetic mastopathy: considerations of a case report. Med Clin (Barc) 2002; 118: 639.

6. Weinstein SP, Conant EF, Orel SG, Lawton TJ, Acs G. Diabetic mastopathy in men: imaging findings in two patients. Radiology 2001; 219: 797-9. nes pueden estar ausentes uno o más hallazgos ${ }^{11}$. También se han descrito proliferaciones fibrosas mamarias benignas en diabéticos no insulinodependientes y en pacientes con otros desórdenes endocrinos particularmente de tiroides ${ }^{13}$.

El conocimiento de la mastopatía diabética y la utilización de la biopsia core para certificar su diagnóstico pueden evitar en las pacientes portadoras de esta patología, un número de biopsias abiertas innecesarias y a veces múltiples, así como reducir el estado de ansiedad que se crea ante un diagnóstico incierto 4 .

7. Shaffrey JK, Askin FB, Gatewood OM, Brem R. Diabetic fibrous mastopathy. Case reports and radiologic-pathologic comelation. Breast J 2000; 6: 414-7.

8. Andrews-Tang D, Diamond AB, Rogers L, Butler D. Diabetic mastopathy: Adjunctive use of ultrasound and utility of core biopsy in diagnosis. Breast J 2000; 6: 183-8.

9. Wong KT, Tse GM, Yang WT. Ultrasound and MR imaging of diabetic mastopathy. Clin Radiol 2002; 57: 730-5.

10. SOler NG, KhaRDori R. Fibrous disease of the breast, thyroids and cheiroarthropathy in type I diabetes mellitus. Lancet 1984; 1: 193-5.

11. Rosen PP. Rosen's Breast Pathology: Inflammatory and Reactive Tumors. New York, LippincottRaven Publishers 1997; 46-9.

12. Tomaszewski JE, BRooks JS, Hicks D, Livolsi VA. Diabetic mastopathy: a distinctive clinicopathologic entity. Hum Pathol 1992; 23: 780-6.

13. Mak CW, Chou CK, Chen SY, Lee PS, Chang JM. Diabetic mastopathy. Br J Radiol 2003; 76: 192-4. 